\title{
O TRABALHO DA ENFERMAGEM NA IMUNIZAÇÃO NO CONTEXTO DA CRISE SANITÁRIA BRASILEIRA
}

\author{
Sonia Acioli' \\ ORCID: 0000-0002-0772-8235
}

Helena Maria Scherlowski Leal David'

ORCID: 0000-0001-8002-6830

Inês Leoneza de Souza"

ORCID: 0000-0001-8280-3306

Maria da Glória Lima"'

ORCID: 0000-0001-6450-9154

Mercedes Neto'

ORCID: 0000-0001-7529-9535

Ricardo de Mattos Russo Rafael'

ORCID: 0000-0003-1315-4271

Sandra Rejane Soares Ferreiralv ORCID: 0000-0003-2742-3700

Universidade do Estado do Rio de Janeiro. Rio de Janeiro, Brasil.

"Universidade Federal do Rio de Janeiro Campus Macaé (UFRJ-Macaé). Macaé, Rio de Janeiro, Brasil.

"'Universidade de Brasília (UnB), Distrito Federal, Brasil.

"Verência de Saúde Comunitária do Grupo Hospitalar Conceição. Porto Alegre, Brasil.

Autora Correspondente: Sonia Acioli

E-mail: soacioli@gmail.com

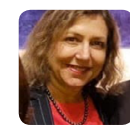

Como citar:

Acioli S, David HMSL, Souza IL, et al. In: Silva TMR,

Lima MG, (Orgs.). Estratégias de vacinação contra a

COVID-19 no Brasil: capacitação de profissionais e discentes de enfermagem. Brasilia, DF: Editora ABen; 2021. $P$ 5-13. (Série enfermagem e pandemias, 6). https://doi.org/10.51234/aben.21.e08.c01

Revisora: Valéria Bertonha Machado. Vice Líder do Laboratório de Estudos em Gestão dos Serviços de SaúdeLABGEST. Departamento de Enfermagem. Faculdade de Ciências da Saúde. Universidade de Brasília.

\section{INTRODUÇÃO}

A situação da pandemia da síndrome respiratória aguda grave 2 (SARS-CoV-2) ou COVID-19 no país desde 2020 continua sem controle, com mais de 600 mil vidas perdidas. A pandemia, enquanto crise sanitária, exacerbou o aprofundamento das desigualdades sociais, expressão das crises social, política e econômica, fruto do modelo neoliberal em curso há muitos anos no país. Desde o golpe parlamentar de 2016, medidas como o congelamento das despesas com saúde e educação, chanceladas pela Emenda Constitucional no 95 (EC95), de dezembro de 2016, aprofundaram a diminuição de financiamento para o Sistema Único de Saúde (SUS), o ensino público, a pesquisa, ciência e tecnologia ${ }^{(1)}$.

Nesse sentido, aprofunda-se a fragilização da Atenção Primária à Saúde (APS) que, apesar da desconstrução sofrida com a Política Nacional de Atenção Básica (PNAB) de 2017, vem demonstrando sua força e importância com o esforço de gestores municipais e estaduais e, especialmente, de trabalhadoras e trabalhadores da saúde na organização da vacinação no país. Apesar da ausência de um plano nacional de vacinação, o que determinou uma campanha de vacinação lenta, sem vacina para toda a população, além da guerra de informações falsas sobre a eficácia e procedência das vacinas, dentre outros aspectos, os profissionais de enfermagem, enfermeiros, técnicos e auxiliares de enfermagem têm assumido papel fundamental no planejamento e desenvolvimento da imunização no país ${ }^{(2)}$.

A Enfermagem brasileira é a maior categoria de profissionais da saúde expostos na linha de frente da atenção à COVID-19 ${ }^{(3)}$. Desde janeiro de 2021, as(os) trabalhadoras(es) de enfermagem, mesmo em um contexto bastante adverso, têm participado diretamente da operacionalização da campanha nacional de vacinação contra a COVID-19, nos 5.570 municípios brasileiros das 27 unidades da federação.

O Brasil aplicou mais de 214 milhões de doses de vacinas contra a COVID-19 até o dia 14 de setembro de $2021^{(4)}$. 
O processo de imunização priorizou grupos de risco, iniciando a vacinação, no primeiro momento, pela população idosa e profissionais de saúde da linha de frente, seguindo, na medida da disponibilidade dos imunobiológicos, pelos grupos mais vulneráveis (comorbidades, população em situação de rua, indígenas, entre outros). Atualmente, a vacinação se volta para as faixas etárias mais jovens. A campanha de vacinação contra a COVID-19 avança de forma heterogênea nos diversos municípios brasileiros, com critérios epidemiológicos diferenciados.

Apesar das baixas remunerações, grandes diferenças salariais entre os profissionais de saúde, exaustão devida às longas jornadas de trabalho, com acúmulo de empregos para garantir a sobrevivência, ambientes físicos inadequados, falta de materiais e equipamentos apropriados, até mesmo adoecimento e alta mortalidade dos profissionais de enfermagem, a Enfermagem brasileira tem desempenhado com competência, comprometimento e protagonismo o papel de cuidar durante a pandemia, nas diferentes fases do processo - acolhimento, rastreio e testagem dos casos suspeitos, cuidados intensivos e reabilitação pós-COVID-19.

$\mathrm{Na}$ APS, as atividades de imunização contra a COVID-19 representam ações extraordinárias às rotinas de imunização realizadas cotidianamente nas unidades de saúde, demandando de todas as instâncias - gestores e vacinadores - a realização de um plano de trabalho que deveria ter sido orientado pelo Programa Nacional de Imunização (PNI), para garantir a efetividade e equidade do processo, bem como a integridade e a proteção específicas de profissionais e usuárias(os) dos serviços de saúde. Embora o Ministério da Saúde (MS) tenha criado, em 10 de dezembro de 2020, o primeiro documento orientador sobre a Campanha Nacional de Vacinação contra a COVID-19, com previsão de quatro fases e definição dos grupos prioritários, sua divulgação ocorreu de forma lenta e pouco esclarecedora para ser de fato o documento condutor de todo o processo nos estados e municípios.

Apesar das diversas crises, em todos os estados e no Distrito Federal tem havido grandes manifestações e movimentos da sociedade civil, dos quais a enfermagem tem participado por meio da Associação Brasileira de Enfermagem (ABEn) e outras entidades da saúde e da enfermagem, reivindicando aceleração no ritmo da vacinação, defesa do auxílio emergencial e valorização da educação e da saúde no país, e, também, manifestando repúdio contra a política econômica do governo federal e sua condução na pandemia causada pelo coronavírus.

Em contexto de tantas demandas, crises e contradições, são inúmeras as experiências de organização e reorganização dos processos de trabalho de enfermeiras(os), técnicas(os) e auxiliares de enfermagem. No entanto, neste capítulo, buscamos refletir sobre o trabalho e as práticas das(os) trabalhadoras(es) de enfermagem no programa de imunizações, as potencialidades e os limites da atuação no contexto da pandemia da COVID-19.

\section{CONTEXTO DAS IMUNIZAÇÕES NO SUS E O TRABALHO DA ENFERMAGEM}

O PNI foi criado no Brasil em 1973 e regulamentado em 1975, com o objetivo de controlar doenças preveníveis por vacinas. Essa implementação na saúde possibilitou o fortalecimento do papel do Ministério da Saúde como responsável pela organização e coordenação das vacinas, que já ocorriam há várias décadas, trazidas ao país desde o século XIX(5).

Vale destacar que as políticas públicas de imunização do Brasil são consideradas referência mundial há muitos anos e, assim como a história da imunização em geral, a história da imunização no Brasil está atrelada à criação da vacina contra a varíola, que, com a Revolta da Vacina em 1904, teve marco na memória e evolução das ações de imunização no país, paralelamente ao que se desenvolvia no âmbito maior - a saúde pública.

O programa se tornou relevante com a implementação do SUS, a partir do qual os municípios se tornaram os executores diretos das ações em saúde, entre elas a vacinação. A partir de então, ocorreu importante aumento da cobertura vacinal, uma vez que todas as Unidades Básicas de Saúde (UBS) passaram a ter acesso às vacinas e a administrá-las ${ }^{(6)}$. Essas vacinações ocorrem, em sua maioria, nas salas de vacinação das UBS, cuja responsabilidade técnica é da(o) enfermeira(o), que é responsável por orientar e prestar assistência em 
condições seguras e de qualidade aos usuários, garantir a manutenção, organização e preservação dos imunobiológicos em condições adequadas, além da administração correta das vacinas, que estão estabelecidas como rotina no calendário vacinal ${ }^{(7)}$. Nessa perspectiva, a(o) enfermeira(o) que trabalha com imunização no SUS tem em sua prática ações que vão muito além de cuidados pontuais relacionados à administração e organização de uma sala de vacinas, assumindo o protagonismo no fomento de estratégias educativas, prospectivas e integrativas, que inserem o cuidado em saúde no contexto da família e da comunidade(8). Também, é responsável por monitorar todos os aspectos técnicos e operacionais, tanto no que se refere à centralização para guarda e distribuição dos imunobiológicos com segurança, quanto à sua conservação nas salas de vacinas. Ainda, a(o) enfermeira(o) está envolvida(o) em ações que dão suporte ao processo de vacinação, como atualizações de procedimentos, técnicas e normas, capacitação de pessoal, supervisão e ações de educação em saúde para a população(9).

As(os) trabalhadoras(es) de enfermagem estão envolvidas(os) no conjunto de ações necessárias para a realização das imunizações nos serviços de saúde da APS, tais como vacinação de rotina, campanhas, ações de bloqueio vacinal e atividades fora da área física da unidade de saúde, como busca de faltosos na comunidade ou vacinação domiciliar, tendo como objetivo ampliar as coberturas vacinais, conferindo proteção individual e coletiva para a população(9).

Cabe destacar que o trabalho da enfermagem nas imunizações segue premissas fundamentais para atingimento dos resultados com a adequada cobertura vacinal, entre elas ${ }^{(9)}$ :

a) a vacinação pode controlar e até erradicar doenças, por isso deve ser realizada de forma que todas as etapas do processo de imunização da população ocorram com segurança e eficácia;

b) de modo geral, no Brasil e no mundo, a imunização das populações depende do trabalho da enfermagem;

c) a enfermagem segue as recomendações científicas sobre vacinação;

d) a enfermagem deve combater os mitos sobre a vacinação, ou seja, as equipes de enfermagem devem estar preparadas para desmentir qualquer fake news, apresentando dados e estudos de fontes científicas; $\mathrm{e}$

e) para alcançar o objetivo de uma imunização coletiva, é imprescindível uma atuação intersetorial.

\section{A RESPONSABILIDADE DOS PROFISSIONAIS DE ENFERMAGEM NO PROCESSO DE IMUNIZAÇÕES E O CONTEXTO DA COVID-19}

No conjunto de atividades desenvolvidas pela enfermagem no processo de imunização, os aspectos operacionais da sala de vacinas merecem atenção especial da(o) enfermeira(o), como coordenadora(or) da equipe de enfermagem, porque elas(es) são essenciais para a administração dos imunobiológicos em conformidade com os padrões recomendados de conservação, armazenagem, indicação clínica e cuidados pré e pós sua aplicação, assegurando que a vacina realmente cumpra com o objetivo de proteger a saúde das pessoas contra as doenças imunopreveníveis ${ }^{(9)}$.

À(o) enfermeira(o) cabe privativamente a supervisão de todo o processo de trabalho com as imunizações, o qual abrange ações como as ora destacadas, especialmente durante a pandemia da COVID-19:

a) organizar e adequar a área física das unidades de saúde e de outros locais (drive thru, posto volante, domicílio, instituições) para a realização da imunização. $A(0)$ enfermeira(o) atua como responsável técnico pelas ações de imunização e, nesse sentido faz a gestão e preparação dos locais onde a prática será executada, de forma a garantir todas as medidas de segurança para evitar a contaminação e a transmissibilidade da COVID-19, garantindo a segurança da população que será imunizada e dos profissionais de enfermagem. É desejável que o ambiente destinado à vacinação tenha local de entrada e saída independentes, permitindo um fluxo contínuo de atendimento às(aos) usuárias(os) e, assim, 
reduzindo riscos de contaminação pela COVID-19. Portanto, esse profissional necessita promover condições no serviço de saúde para higiene simples das mãos, como a disponibilidade de lavatório/ pia com dispensador de sabonete líquido ou com álcool gel a 70\%, suporte para papel toalha, papel toalha, lixeira com tampa;

b) promover o adequado manuseio, conservação, preparo e administração do imunizante, bem como o registro das doses aplicadas, no sistema de informação do PNI, descarte dos resíduos, avaliação e monitoramento epidemiológico;

c) orientar o uso adequado dos EPIs no processo de vacinação, como: máscara cirúrgica (que deve ser trocada a cada duas horas e sempre que estiver úmida ou suja); protetor facial (face shield) ou óculos de proteção, e avental descartável para uso diário (um por dia) ou avental de tecido, que deve ser higienizado diariamente no ambiente do serviço, evitando que o profissional leve o avental para a sua residência; luvas de procedimento somente para indicações específicas, como vacinadores com lesões cutâneas ou raras situações que envolvam contato com fluidos corporais do paciente ou que ele apresente lesões de pele no local da aplicação. Se usadas, devem ser trocadas entre os pacientes, associadas à adequada higienização das mãos;

d) orientar que o respirador PFF2/N95 esteja recomendado para ambientes sem ventilação/circulação de ar adequada, para uso com pacientes institucionalizadas(os) ou confinadas(os), como nas Instituições de Longa Permanência de Idosos (ILPI) e estabelecimentos prisionais, ou que apresentem risco de aerossóis;

e) orientar a higienização das mãos: a cada vacinação, com álcool gel 70\%, e a cada cinco vacinações, a lavagem com água e sabão;

f) pactuar com o gestor local a necessidade de implantar medidas sanitárias preventivas para a população, com alertas visuais (cartazes, placas, pôsteres, entre outros), informações sobre o uso permanente de máscaras, os principais sinais e sintomas da COVID-19, a forma correta para a higiene das mãos com água e sabonete líquido ou com álcool em gel a $70 \%$ e sobre higiene respiratória/etiqueta da tosse. Também, é fundamental a identificação no solo de pontos que garantam o distanciamento social na entrada dos serviços de saúde e em locais estratégicos;

g) solicitar a organização de espaço/sala para reações adversas na unidade de saúde e outros pontos de vacinação, a fim de estabelecer articulação com uma equipe de resposta rápida nos casos de intercorrências;

h) prever/realizar articulação intersetorial para prevenção de tumulto no local de vacinação (preocupação com falta de doses, atos antivacina, entre outros), invasão e roubo;

i) organizar todas as etapas do processo de contato com a(o) usuária(o) para imunização. Os procedimentos de acolhimento devem ser realizados na entrada do serviço de saúde, antes do ingresso da(o) usuária(o) no ambiente dedicado à vacinação. Nesse sentido, os serviços de saúde devem garantir e considerar a estimativa da demanda diária de usuárias(os), o número de equipes de resposta rápida (constituídas de forma multidisciplinar) para o atendimento oportuno e célere, caso ocorra alguma reação adversa após aplicação da vacina;

j) realizar educação em saúde com a população e orientar o uso permanente de máscara cirúrgica/tecido. Caso a máscara apresente umidade, uso prolongado ou sujidade, sugere-se que a unidade forneça uma máscara cirúrgica à pessoa e que, durante o procedimento de vacinação, a pessoa vire o rosto para o lado contrário ao que será vacinado, mantendo o máximo de distanciamento da(o) vacinadora(or);

k) solicitar à gestão que os espaços de "sala de espera" sejam dotados de iluminação (natural ou artificial), temperatura, umidade e ventilação natural suficientes para o desempenho das atividades em condições adequadas pela equipe de saúde e mitigação dos riscos de transmissão do vírus;

I) garantir agendamento ou horário especial para as(os) idosas(os) (sem misturar com outro tipo de público), realizar vacinação domiciliar às pessoas acamadas ou restritas no domicílio; 
m) prever pessoal de apoio/voluntários para organizar a fila e monitorar o espaçamento de segurança entre as(os) usuários(as) de, no mínimo, 1,0m de distância, a utilização contínua de máscara pelas(os) usuárias(os) durante todo o período de permanência no ambiente, e a organização do fluxo contínuo aos ambientes de registro de dados da(o) usuária(o) / escuta inicial, chegada com segurança até a administração de vacina e saída do ambiente de vacinação, evitando aglomerações em qualquer etapa do processo.

Pode-se verificar que o quantitativo de atividades que precisam ser realizadas antes, durante e após a aplicação das vacinas, é enorme e exige uma postura vigilante e de constante atenção, o que tem gerado estresse às(aos) trabalhadoras de enfermagem, já que não estão envolvidas(os) exclusivamente com a campanha de imunização para a COVID-19. Essa ação se soma às diversas atividades desenvolvidas nas UBS, sem o correspondente aumento do número de profissionais em muitos municípios.

\section{DESAFIOS IMPOSTOS AO PROCESSO DE TRABALHO DAS(OS) TRABALHADORAS(ES) DE ENFERMAGEM NA CAMPANHA DE IMUNIZAÇÃO CONTRA A COVID-19}

Literalmente, tendo estado nas mãos da enfermagem o maior recurso de esperança para controle da pandemia e não tendo havido tempo de preparo suficiente para lidar com tamanha responsabilidade e pressão, poder-se-ia aventar a possibilidade de ocorrência de problemas na administração das vacinas ou alguma conduta inadequada. Acrescenta-se a esse contexto que a enfermagem tem trabalhado em condições adversas, tendo que aplicar vacinas novas e de diferentes tipos no mesmo espaço de imunização, com informações e protocolos de orientação da administração que mudam diariamente, estabelecidos no decorrer do processo de vacinação e de forma diferente em cada município.

Destaca-se que a enfermagem há tempos é submetida a processos de trabalho desgastantes e sem o suporte adequado, em termos de remuneração, equipamentos e jornada de trabalho, conforme citado anteriormente. Nesse contexto, ocorreram relatos nas redes sociais e nos meios jornalísticos sobre a aplicação das vacinas contra a COVID-19 de forma errônea e até mesmo de não-aplicação, o que configura desvio do propósito, falta de ética e, porque não dizer, ato criminoso, que teriam sido realizados por profissionais da enfermagem. Entretanto, as denúncias sobre os problemas na administração da vacina contra a COVID-19, de acordo com o Cofen ${ }^{(10)}$, representam uma ínfima fração do contingente vacinado e, mesmo assim, todos estão sendo investigados, reforçando o compromisso ético da profissão com a saúde da população.

A crise sanitária evidenciou problemas previamente existentes no processo de trabalho da enfermagem e das equipes de APS. A organização e supervisão da sala de vacina em uma UBS é de responsabilidade da(o) enfermeira(o), porém, diante de tantas atividades atribuídas a tais profissionais, em diversas UBS as(os) técnicas(os) e auxiliares de enfermagem acabam assumindo sozinhas(os) o trabalho de vacinação.

A ausência da(o) enfermeira(o) na sala de vacinas pode fazer com que as(os) técnicas(os) e auxiliares de enfermagem fiquem sem supervisão para administrar os imunobiológicos e realizar as orientações necessárias (efeitos adversos, aprazamentos, doença que motivou a imunização etc.). Saliente-se que a ausência da(o) enfermeira(o) na sala de vacinas não é ato de responsabilidade apenas individual, mas está relacionado à gestão dos serviços de saúde, que nem sempre dispõe do quantitativo suficiente de profissionais para dar conta das inúmeras demandas do serviço. Esta ausência decorre do dimensionamento inadequado das equipes de imunização (porte do serviço de saúde e tamanho da população adscrita ao serviço); sobrecarga de trabalho na UBS com o atendimento de porta de entrada para pessoas com suspeita de COVID-19, outros problemas de saúde, imunização de rotina e imunização para a COVID-19; número reduzido de profissionais capacitados para atuar em sala de imunização e falta de capacitação específica para a vacinação contra a COVID-19 nas populações prioritárias. 
Outro desafio importante enfrentado na campanha de imunização da COVID-19 é a desinformação da população sobre a doença e sobre a imunização, agravadas por fake news, que influenciaram o comportamento contrário à imunização. Existe um movimento antivacina anterior à pandemia da COVID-19 que leva a população a um comportamento de indecisão/retardo na utilização das vacinas, induzindo atitudes de risco, não só à saúde individual do não vacinado, mas de todos à sua volta. Pode-se supor que a possível baixa adesão à vacinação contra a COVID-19 e/ou à segunda dose do imunizante esteja relacionada a vários fatores, entre eles a existência de diferentes eficácias dos imunobiológicos disponíveis no Brasil, o negacionismo de parte da população com relação à realidade ou gravidade do vírus, as fake news sobre vacinas e a oferta insuficiente das vacinas à população.

Acrescenta-se aos desafios citados a falta da estrutura física adequada para se realizar a vacinação, conforme preconizado pelo Ministério da Saúde, sendo imprescindível um olhar crítico voltado para a estrutura física do ambiente e para as condições de trabalho desses profissionais. Os aspectos operacionais do ambiente de vacinação são essenciais para a administração dos imunobiológicos de acordo com os padrões recomendados de conservação, armazenagem, indicação clínica e cuidados pré e pós a aplicação, assegurando que a vacina realmente cumpra com o objetivo de proteger a saúde das pessoas contra as doenças imunopreveníveis.

Outro problema evidenciado nas campanhas de imunização para a COVID-19 foi a ausência de uma concepção política de intersetorialidade no planejamento de ações de enfrentamento para problemas complexos, como a crise sanitária ocasionada pela COVID-19 e seus efeitos. Esse problema foi agravado pela ineficiência governamental para definir, de forma conjunta, rápida e efetiva, políticas públicas e de saúde para o enfrentamento da emergência sanitária.

\section{CONTEXTO DE CRISE SOCIAL E SANITÁRIA PARA O ENFRENTAMENTO DA PANDEMIA}

Um dos legados da atual emergência sanitária, se é que se pode assim dizer, estará intimamente relacionado ao futuro do SUS como política pública essencial para a sociedade brasileira e o reconhecimento político do protagonismo dos profissionais de saúde, movimentos sociais e da sociedade civil no processo de resistência e enfrentamento das crises que envolvem a pandemia da COVID-19.

Nessa perspectiva, há dois caminhos a serem escolhidos: um que mantém e reafirma a saúde como um direito e dever do Estado, aprovado da Constituição Federal de $1988^{(13)}$, com a instituição do SUS, de caráter universal, descentralizado e hierarquizado, como segundo nível de atenção e complexidade da assistência aos indivíduos e grupos, definidos nas Leis $\mathrm{n}^{\circ} 8080 / 1990^{(11)}$ e $\mathrm{n}^{\circ} 8142 / 1990^{(12)}$, e, ainda, como prestador de serviço estatal, com base forte na seguridade social. O outro caminho é o que redireciona o modelo para atender à saúde definida a partir da doença, entendida como uma mercadoria, com seu forte apelo em franca expansão apoiado, infelizmente, pela brecha no art. 199 $9^{(13)}$, a participação complementar de instituições privadas, forma complementar do SUS.

No contexto de pandemia da COVID-19, ao se olhar o arcabouço normativo do SUS, tem-se o indicativo das ações de articulação entre os estados da federação e seus respectivos municípios como o ponto chave para a sustentabilidade da rede de serviços no SUS. Dessa forma, é fundamental a aplicação de investimentos financeiros para pessoal, material e logística, visando a uma atuação cada vez mais forte da APS como nível de atenção à saúde, para se vencer a pandemia. Nessa direção, é imprescindível reforçar que o papel dos gestores no combate à pandemia da COVID-19 passa por fornecer informações e orientações técnicas sobre a execução de políticas de saúde e pelo compartilhamento de boas práticas de gestão, tanto no nível municipal quanto no estadual ${ }^{(14)}$.

O Brasil possui um dos maiores sistemas universais de saúde do mundo, o SUS, ancorado em uma extensa rede de serviços, especialmente de APS. No entanto, constata-se um desmonte em curso, que implica a perda, dia após dia, da sua robustez. É um sistema cronicamente subfinanciado desde sua implantação, com UBS que 
procuram responder ao contexto de vigilância em saúde e de emergência pública, mesmo sem aporte de recursos suficientes, por que somos e continuamos a ser um dos países que menos investe em saúde per capita ${ }^{(15)}$.

Podem-se citar como limitações e desafios no enfrentamento da pandemia as dificuldades do Ministério da Saúde em manter um comando único, com as Secretarias Estaduais e Municipais de Saúde adotando condutas não alinhadas às orientações e diretrizes ministeriais, o que piora significativamente o cenário de crise no sistema de saúde no Brasil. Não por acaso, com o limite do teto de gastos, o SUS agoniza e se distancia de forma acentuada dos dispositivos de sustentabilidade dos serviços e dos servidores, em escala assustadora para a realidade e tristeza do povo brasileiro.

As competências das três instâncias do SUS foram definidas para assumir papel de atores estratégicos do sistema. Como exemplo, o Município deve prover os serviços de vigilância epidemiológica, sanitária e ambiental; de alimentação e nutrição; de saneamento básico e saúde ocupacional; de controle e fiscalização dos procedimentos dos serviços privados de saúde. O Estado é responsável pelas ações de saúde, planejamento e controle do SUS e por estimular o trabalho em rede e/ou áreas por regiões, tudo dentro da sua esfera de atuação e regulação. A União deve normatizar o conjunto de ações de promoção, proteção e recuperação da saúde, identificando riscos e necessidades nas diferentes regiões ${ }^{(16)}$.

Na perspectiva de um sistema de saúde universal e público, as ações de controle e enfrentamento da pandemia não prescindem da mobilização de organizações populares e comunitárias. Num contexto de desemprego crescente e precarização do trabalho da população residente nas áreas periféricas das capitais brasileiras, logo se tornou claro que "ficar em casa" não é uma opção para toda a classe trabalhadora brasileira.

Nas favelas brasileiras, os processos de organização comunitária, que já existiam há décadas, deram origem a novas formas de mobilização voltadas para o enfrentamento da pandemia e suas consequências, expondo o desafio de buscar recursos para garantir a alimentação daqueles que já sofriam ou passaram a sofrer com o desemprego, o que se somou à impossibilidade de contar com o apoio da escola na alimentação e espaço para as crianças. A ocorrência de casos de COVID-19 nas famílias que vivem em moradias exíguas e com acesso deficiente à água determinou a rápida organização de grupos comunitários, cujas atividades foram se tornando mais e mais visíveis nas mídias sociais. Pode-se afirmar que esses grupos, que inclusive realizam levantamentos de dados epidemiológicos, agiram de forma mais rápida e efetiva do que o poder público, que se limitou à corrida pela oferta de leitos de terapia intensiva, deixando o nível da Atenção Primária - aquele que é justamente o que se responsabiliza pela produção do cuidado no território - em segundo plano(2). Os setores acadêmicos, em especial os das Instituições de Ensino Superior (IES), desenvolveram iniciativas rápidas e diversas, se constituindo como recurso para somar às redes sociais solidárias, com adaptações no ensino, a aceleração e o incremento de pesquisas básicas e aplicadas, ofertas de extensão. Essas iniciativas fortaleceram as ações de apoio à população e à mobilização nos territórios, ao enfrentamento e prevenção da COVID-19. Várias dessas experiências foram evidenciadas já nos primeiros meses da pandemia, em IES, como por exemplo, no Boletim da Unicamp - Universidade Estadual de Campinas ${ }^{(17)}$.

Assim como as IES, as sociedades científicas se uniram para mobilizações com foco na qualidade e atualidade das informações científicas a serem disseminadas. Por um lado, a pandemia acirrou o que se tem denominado de guerra de narrativas - muito embora quando se trata de evidências científicas fala-se de elementos concretos que se mostram como a melhor resposta disponível no momento, não se tratando apenas de um discurso. Por outro lado, a enxurrada informacional trouxe à luz questões como a relação entre os campos científico, político e religioso, e a circulação de meias verdades, ou fake news, mostrou ser mais que um ato de irresponsabilidade, podendo se constituir um crime contra o processo civilizatório(18). É preciso ir além do aspecto emergencial das ações de mobilização social e comunitária, e resgatar conceitos como o de construção partilhada do conhecimento, ou terceiro conhecimento ${ }^{(19)}$ e a radicalidade da base educativa crítica, legado do pensamento Freireano. O que esse diálogo e ações comuns entre os setores acadêmicos e comunitários mostram é que certamente é um equívoco avaliar que as classes populares, pobres e periféricas 
brasileiras estão paradas ou não são capazes de produzir respostas potentes aos desafios cotidianos e às emergências globais.

\section{CONSIDERAÇÕES FINAIS}

Os profissionais de enfermagem, sob a coordenação da(o) enfermeira(o), exercem um importante papel no tocante às imunizações, tanto na gestão dos imunobiológicos, em âmbito nacional, estadual e municipal, quanto na aplicação dos imunobiológicos nas UBS e Centros de Referência em Imunobiológicos Especiais (CRIES).

A responsabilidade de lidar com os imunobiológicos é imensa e, nem sempre, as(os) trabalhadoras(es) de enfermagem possuem as condições estruturais adequadas para o trabalho nas UBS. Muitas vezes, também faltam ofertas de processos de educação permanente voltados à imunização para a qualificação e atualização da equipe. O número insuficiente de profissionais e a sobrecarga no trabalho precisam ser revistos com urgência, para se evitar prejuízos para as(os) trabalhadoras(es) e usuárias(os). É imprescindível que os municípios ofereçam condições adequadas para o trabalho e que durante a emergência sanitária ampliem o quantitativo de trabalhadoras(es) em enfermagem na APS, visando ao atendimento das necessidades específicas para enfrentamento da pandemia da COVID-19.

Em relação à gestão em saúde, há inúmeros desafios para além dos problemas evidenciados na assistência ao combate à COVID-19, como a escassez de recursos, falta de materiais e inexperiência dos profissionais diante da pandemia, acrescentando ainda os muitos desafios relacionados ao pós-pandemia. Por isso, entende-se a APS como estratégica na reorganização da atenção à saúde.

Apesar dos inúmeros limites dados pela crise política e institucional e, especialmente pela crise sanitária, com aprofundamento dos processos de precarização do trabalho em saúde, e vulnerabilização de grupos sociais, faz-se necessário reafirmar princípios e lutar na defesa da vida, das boas práticas de cuidado e da melhoria da assistência à saúde da população.

\section{REFERÊNCIAS}

1. Souza IL, Oliveira AS, Rafael RMR, Rodrigues PHA. A atenção primária à saúde na pós-pandemia e a prática dos profissionais de enfermagem. In: Teodósio SS-CS, Leandro, SS, (Orgs.). Enfermagem na atenção básica no contexto da covid-19. Vol. 1. Brasília, DF: ABEn; 2020. p. 11-17. (Série enfermagem e pandemias, 3). https://doi.org/10.51234/aben.20.e03.c02

2. David HMSL, Acioli S, Silva MRF, Bonetti OP, Passos H. Pandemics, crisis conjunctures, and professional practices: what is the role of nursing with regard to covid-19?. Rev Gaúcha Enferm. 2020;42(spe):e20200254. https://doi. org/10.1590/1983-1447.2021.20190254

3. Conselho Federal de Enfermagem (BR). Enfermagem em números [Internet]. Brasília, DF: Cofen; 2015[cited 2021 Oct 26]. Available from: http://www.cofen.gov.br/enfermagem-em-numeros

4. Mapa da vacinação contra covid-19 no Brasil. G1 [Internet]. 2021 Sept 13[cited 2021 Sept 14]. Available from: https:// especiais.g1.globo.com/bemestar/vacina/2021/mapa-brasil-vacina-covid/

5. Sato APS. Programa nacional de imunização: sistema informatizado como opção a novos desafios. Rev Saude Publica. 2015:49:39. https://doi.org/10.1590/S0034-8910.2015049005925

6. Domingues CMAS, Woycicki JR, Rezende KS, Henriques CMP. Programa nacional de imunização: a política de introdução de novas vacinas. Rev Eletron Gestao Saude. 2015[cited 2021 Oct 26];6(suppl 4):3250-74. Available from: https://periodicos. unb.br/index.php/rgs/article/view/3331

7. Horbe BP, Santini TP, Adames N, Haeffner LSB, Naujorks AA, Backes DS. Public imunization sistem versus private immunization sistem: comparisons and attribution of nursing. Res Soc Dev. 2020;9(5):e169953355. https://doi.org/10.334448/rsd-v9i5.3355

8. Silva CP, Dias MSA, Rodrigues AB. Práxis educativa em saúde dos enfermeiros da estratégia saúde da família. Cienc Saude Colet. 2009:14(suppl 1):1453-62. https://doi.org/10.1590/s1413-81232009000800018 
9. Périco LAD, Wiederkehr PC. Imunizações. In: Ferreira SRS, Périco LAD, Dias VRFG. Atuação do enfermeiro na atenção primária à saúde. Rio de Janeiro: Atheneu; 2017. p. 273-296.

10. Conselho Federal de Enfermagem (BR). Cofen apura denúncias sobre a vacinação contra covid-19 [Internet]. Brasília, DF: 6 Apr 2021 [cite 2021 Oct 26]. Available from: http://www.cofen.gov.br/cofen-apura-denuncias-sobre-a-vacinacao-contracovid-19_86215.html

11. Presidência da República (BR). Lei 8.080, de 19 de setembro de 1990. Dispõe sobre as condições para a promoção, proteção e recuperação da saúde, a organização e o funcionamento dos serviços correspondentes e dá outras providências [Internet]. Brasília, DF: PR; 1990[cited 2021 Sep 14]. Available from: http://www.planalto.gov.br/ccivil_03/leis/ I8080.htm

12. Presidência da República (BR). Lei 8.142, de 28 de dezembro de 1990. Dispõe sobre a participação da comunidade na gestão do Sistema Único de Saúde (SUS) e sobre as transferências intergovernamentais de recursos financeiros na área da saúde e dá outras providências [Internet]. Brasília, DF: PR; 1990[cited 2021 Sep 14]. Available from: http://www.planalto. gov.br/ccivil_03/leis/l8142.htm

13. Brasil. Constituição da República Federativa do Brasil de 1988 [Internet]. Brasília, DF: Senado; 1988[cited 2021 Sep 14]. Available from: http://www.planalto.gov.br/ccivil_03/constituicao/constituicao.htm

14. Tribunal de Contas da União. TCU+Cidades: o papel do gestor local no combate à pandemia da covid-19 [Internet]. Brasília, DF: 2021 Apr 19[cited 2021 Sep 14]. Available from: https://www.youtube.com/watch?v=RAUq3EEJmso

15. Conselho Regional de Farmácia (RJ). Brasil é um dos países que menos investem em saúde pública [Internet]. Rio de Janeiro: CRFRJ; 2015 Jun 26[cited 2021 Sep 14]. Available from: https://crf-rj.org.br/noticias/1102-brasil-e-um-dos-paisesque-menos-investem-em-saude-publica.html

16. Gondim RO, Grabois V, Mendes Jr WV, organizadores. Qualificação de gestores do SUS [Internet]. Rio de Janeiro: EAD Ensp; 2009[cited 2021 Sep 14]. Available from: https://biblioteca.univap.br/dados/00002d/00002dfd.pdf

17. UNICAMP. Universidades brasileiras e Covid-19: fortalecendo os laços com a sociedade. Boletim [Internet]. [Campinas]: Unicamp; 23 Apr 2020[cited 2021 Sep 14]. Available from: https://www.unicamp.br/unicamp/coronavirus/ universidades-brasileiras-e-covid-19-fortalecendo-os-lacos-com-sociedade

18. David HMSL, Martínez-Riera JR. Fake news and small truths: a reflection on the political competence of nurses. Texto Contexto Enferm. 2020;29:e20190224. https://doi.org/10.1590/1980-265X-TCE-2019-0224

19. Acioli S, Azevedo DCV, Pascoal R, Ferreira VS, Silva ABP. Reflexões sobre a construção compartilhada do conhecimento em saúde na localidade do Alto Simão / Vila Isabel, RJ. Interagir [Internet]. 2002[cited 2021 Sep 10];(2):17-22. Available from: https://www.e-publicacoes.uerj.br/index.php/interagir/article/download/18651/13608 